Earlier studies have led to a hypothesis of an increased risk of breast cancer after exposure to organic solvents. This study aimed to investigate if exposure to various chemicals in the workplace was associated with an increased risk of breast cancer.

The study comprised 14119 women born 1923-1950 and living in Malmö city, Sweden, who were enrolled for a prospective population cohort study between 1991 and 1996. Oc cupational exposure to various chemicals was assessed from a job-exposure matrix. Individual data on other breast cancer risk factors (age, parity, age at first pregnancy, breastfeeding, hormonal replacement therapy, physical activity, alcohol consumption, height and BMI) were collected at baseline and used for confounding control. Diagnoses of invasive breast cancer were obtained from the Swedish Cancer Registry until end of follow-up at 2013-12-31.

Women exposed to any type of chemical (organic solvents, pesticides, fumes or oil mist) for more than 10 years (66 cases) showed an increased adjusted breast cancer risk, $\mathrm{HR}=1.43, \quad(95 \%$ CI 1.10-1.85), and the risk correlated positively with duration of exposure. Investigation of risk in association with organic solvents showed a small and non-significantly elevated risk after $>10$ years of exposure, $\mathrm{HR}=1.18$ (95\% CI 0.81-1.71). The risk tended to be higher, but not significantly elevated, after $>10$ year of exposure than after 1-10 years of exposure for most of the studied chemicals. More than 10 years of exposure to diesel exhaust was associated with an increased risk (adjusted $\mathrm{HR}=1.69$, 95\% CI 1.012.82).

Occupational exposure to chemicals in general was associated with an elevated risk of postmenopausal breast cancer, but the study gives no firm support for the hypothesis of an association with exposure to organic solvents. An elevated risk after $>10$ years of exposure to diesel exhaust was an unexpected finding that needs to be investigated further.

\section{A.5 MORTALITY AND RISK BEHAVIOURS IN SPANISH FEMALE FARMERS}

${ }^{1}$ Guanlan Zhao*, ${ }^{*}$ Elena Ronda, ${ }^{2}$ Gregorio Barrio, ${ }^{3}$ Enrique Regidor. ${ }^{1}$ University Of Alicante, Alicante, Spain; ${ }^{2}$ National School of Public Health, Instituto de Salud Carlos III, Madrid, Spain; ${ }^{3}$ Complutense University of Madrid, Madrid, Spain

\subsection{6/OEM-2019-EPI.140}

Introduction Numerous epidemiological studies have been conducted among farmers, but very few of them have included female farmers. Therefore, the health status of female farmers remains understudied. Our aim is to compare the mortality rates and the prevalence of risk behaviours in a cohort of female farmers and female non-farmers in Spain.

Methods The cohort study followed 5,919,413 female workers in Spain from 2001 to 2011. Participants aged 20-64 years were employed in 2001. The study subjects were grouped as farmers and non-farmers The prevalence of risk behaviours was calculated from the 2001 National Health Survey for 5573 employed women. For each cause of death, we estimated the age-standardized mortality rates per 100000 person-years of follow-up, the ratio of age-standardized mortality rates with 95\% confidence intervals (CI), and for each risk behaviours age-standardized prevalence ratio in female farmers compared female non-farmers.

Results The mortality rate ratio of female farmers compared to the female non-farmers was 1.00 (95\% CI 0.96-1.05). For most these cause of deaths no significant differences were observed in the mortality rates between both groups, except in mortality from lung cancer, chronic lower respiratory disease, transport accidents and suicide, which mortality rate ratios were $0.56(0.46-0.67), 0.44(0.23-0.87), 1.47$ (1.16$1.86)$ and $1.41(1.10-1.81)$, respectively. The prevalence of smoking and excessive alcohol consumption was lower in female farmers.

Discussion We found a decreased in the mortality ratio for tobacco-related cancers. Exposure to psychosocial risk factors could be the reason of higher mortality of external causes of death of Spanish female farmers.

\section{A.6 MORTALITY EXPERIENCE OF IN WOMEN IN SOUTH AFRICAN MINING: $2013-2015$}

${ }^{1}$ Kerry Wilson*, ${ }^{1,3}$ Tahira Kootbodien, ${ }^{1,4}$ Nisha Naicker. ${ }^{1}$ National Institute for Occupational Health, Johannesburg, South Africa; ${ }^{2}$ University of the Witwatersrand, Johannesburg, South Africa; ${ }^{3}$ Universsity of Cape Town, Cape Town, South Africa; ${ }^{4}$ Univeristy of Johannesburg, Johannesburg, South Africa

\subsection{6/OEM-2019-EPI.141}

Mining is a high-risk industry with both continued accidents and occupational disease, despite controls introduced in the industry. In this study, we looked at the sex differences in mortality between male and female miners in South Africa.

Methods The use of vital registration data for monitoring mortality in miners has largely been unexplored in South Africa. Statistics South Africa provides data from 2013 to 2015 which was used in students-t-tests along with proportion tests to investigate differences between death in all women and women miners along with differences in deaths in male miners and women miners. Multiple logistic regression analysis was performed to calculate mortality odds ratios (MORs) for the underlying cause of death in these groups, with adjustments for age, education level, province of death and smoking status.

Results Of the 8769 deaths recorded with occupation miner ion the years 2013-2015, only 5.7\% were in females. Significant differences between all women and women miners were found in age at death (58.8 vs 47.8 ), no 1 cause of death (illdefined vs $\mathrm{TB}$ ) and education (43.6\% vs 63.6\%). MORs were significantly increased in women miners for TB, HIV and external causes of death compared to all women while being protected from lifestyle and chronic diseases. Women miners compared to male miners had increased odds of HIV death and lifestyle diseases but a similar risk of external causes of death.

Discussion Women miners appear to die at significantly younger ages than both male miners and other women despite a higher level of education. This may be due to the increased mortality due to HIV and external causes of death. Thus increased controls are required on mines to protect the health of women miners. 